\title{
Stability Analysis of Linear Discrete-Time Systems with Interval Delay: A Delay-Partitioning Approach
}

\author{
Priyanka Kokil, Haranath Kar, and V. Krishna Rao Kandanvli \\ Department of Electronics and Communication Engineering, Motilal Nehru National Institute of \\ Technology, Allahabad 211004, India \\ Correspondence should be addressed to Priyanka Kokil, kokilnit@gmail.com
}

Received 7 September 2011; Accepted 16 October 2011

Academic Editors: C.-H. Lien, F. Tadeo, and Q. Zhang

Copyright ( $) 2011$ Priyanka Kokil et al. This is an open access article distributed under the Creative Commons Attribution License, which permits unrestricted use, distribution, and reproduction in any medium, provided the original work is properly cited.

This paper considers the problem of asymptotic stability of linear discrete-time systems with interval-like time-varying delay in the state. By using a delay partitioning-based Lyapunov functional, a new criterion for the asymptotic stability of such systems is proposed in terms of linear matrix inequalities (LMIs). The proposed stability condition depends on both the size of delay and partition size. The presented approach is compared with previously reported approaches.

\section{Introduction}

Mathematical models with time delays are frequently encountered in various physical, industrial, and engineering systems due to measurement and computational delays, transmission and transport lags. During the last two decades, there has emerged a considerable interest in the system theoretic problems of time-delay systems. In many applications, time delays must be taken into account in a realistic system design, for instance, chemical processes, thermal processes, echo cancellation, local loop equalization, multipath propagation in mobile communication, array signal processing, congestion analysis and control in highspeed networks, neural networks and long transmission line in pneumatic systems [1-6]. The presence of time delays may result in instability of the designed systems. An excellent survey on the stability results of time-delay systems has been presented in [7]. According to dependence of delay, the available stability criteria are generally classified into two types: delaydependent criteria and delay-independent criteria. Delay-dependent stability criteria generally lead to less conservative results than delay-independent ones, especially if the size of time delay is small [8-12].

Many publications relating to the delay-dependent stability analysis of continuous time-delay systems have appeared (see, e.g., [4, 12-21] and the references cited therein). 
In contrast, little effort has been made for studying the problem of stability of discrete timedelay systems. Utilizing Lyapunov functional method, several delay-dependent stability criteria for discrete-time systems have been reported in the literature [2, 22-27]. By employing novel techniques to estimate the forward difference of Lyapunov functional, stability criteria for linear discrete-time systems with interval-like time-varying delays have been proposed in [26]. The criteria proposed in [26] are less conservative with smaller numerical complexity than [22, 28-31]. The delay partitioning technique has been efficiently utilized in [32-36] to the stability analysis of systems with time-varying delays. Recently, by introducing freeweighting matrices and adopting the concept of delay partitioning, improved stability criteria have been established in [37]. The criteria reported in [37] are not only dependent on the delay but also dependent on the partitioning size. Though the approach in [37] provides less conservative results than $[22,29]$, it would lead to heavier computational burden and more complicated synthesis procedure.

Motivated by these developments, this paper studies the problem of stability analysis of linear discrete-time system with interval-like time-varying delay in the state. The paper is organized as follows. Section 2 introduces model description and preliminaries. By utilizing the delay partitioning idea of [37], a new linear-matrix-inequality- (LMI-) based criterion for the asymptotic stability of discrete-time state-delayed systems is proposed in Section 3. The proposed criterion depends on the size of delay as well as partition size. An example highlighting the usefulness of the presented criterion is given in Section 4.

\section{Model Description and Preliminaries}

The following notations are used throughout the paper:

$\mathbf{R}^{p \times q}$ : set of $p \times q$ real matrices,

$\mathbf{R}^{p}$ : set of $p \times 1$ real vectors,

0: null matrix or null vector of appropriate dimension; the orders are specified in subscripts as the need arises,

$\mathbf{I}_{p}: p \times p$ identity matrix,

$\mathbf{B}^{T}$ : transpose of the matrix (or vector) $\mathbf{B}$,

B $>\mathbf{0}: \mathbf{B}$ is positive-definite symmetric matrix,

$\mathbf{B}<\mathbf{0}: \mathbf{B}$ is negative-definite symmetric matrix.

In this paper, we consider a linear, autonomous, multivariable discrete-time system with interval-like time-varying delay in the state. Specifically, the system under consideration is represented by the difference equation:

$$
\begin{gathered}
\mathbf{x}(k+1)=\mathbf{A} \mathbf{x}(k)+\mathbf{A}_{1} \mathbf{x}(k-d(k)), \\
\mathbf{x}(k)=\boldsymbol{\phi}(k), \quad k=-h_{2},-h_{2}+1, \ldots, 0,
\end{gathered}
$$


where $\mathbf{x}(k) \in \mathbf{R}^{n}$ is the system state vector, $\mathbf{A}$ and $\mathbf{A}_{1}$ are constant matrices with appropriate dimensions, and $d(k)$ is a positive integer representing interval-like time-varying delay satisfying

$$
1 \leq h_{1} \leq d(k) \leq h_{2}
$$

where $h_{1}$ and $h_{2}$ are known positive integers representing the lower and upper delay bounds, respectively, and $\phi(k)$ is an initial value at time $k$. Let the lower bound of the delay $h_{1}$ be divided into $m$ number of partitions such that

$$
h_{1}=\tau m,
$$

where $\tau$ is an integer representing partition size.

Definition 2.1. The equilibrium state $\mathbf{x}_{e}=\mathbf{0}$ of the system (2.1a) and (2.1b)-(2.3) is asymptotically stable if, for any $\varepsilon>0$, there exists $\delta>0$ such that if $\|\boldsymbol{\phi}(k)\|<\delta, k=-h_{2},-h_{2}+1, \ldots, 0$, then $\|\mathbf{x}(k)\|<\varepsilon$, for every $k \geq 0$ and $\lim _{k \rightarrow \infty} \mathbf{x}(k)=\mathbf{0}$.

Lemma 2.2 (see [30]). For any positive definite matrix $\mathbf{W} \in \mathbf{R}^{n \times n}$, two positive integers $r$ and $r_{0}$ satisfying $r \geq r_{0} \geq 1$, and vector function $\mathbf{x}(i) \in \mathbf{R}^{n}$, one has

$$
\left(\sum_{i=r_{0}}^{r} \mathbf{x}(i)\right)^{T} \mathbf{W}\left(\sum_{i=r_{0}}^{r} \mathbf{x}(i)\right) \leq\left(r-r_{0}+1\right) \sum_{i=r_{0}}^{r} \mathbf{x}^{T}(i) \mathbf{W} \mathbf{x}(i) .
$$

\section{Main Result}

In this section, an LMI-based criterion for the asymptotic stability of system (2.1a) and (2.1b)(2.3) is established. The main result may be stated as follows.

Theorem 3.1. For given positive integers $\tau, m$, and $h_{2}$, the system described by (2.1a) and (2.1b)(2.3) is asymptotically stable if there exist real matrices $\mathbf{P}=\mathbf{P}^{T}>\mathbf{0}, \mathbf{Q}_{i}=\mathbf{Q}_{i}{ }^{T}>\mathbf{0}(i=1,2,3)$, $\mathbf{Z}_{i}=\mathbf{Z}_{i}^{T}>\mathbf{0}(i=1,2), \mathbf{X}, \mathbf{Y}$ and $\mathbf{W}$ such that

$$
\left[\begin{array}{ccc}
-\mathbf{Z}_{2} & \mathbf{Y}^{T} & \mathbf{X} \\
\mathbf{Y} & \mathbf{\Psi} & \mathbf{W} \\
\mathbf{X}^{T} & \mathbf{W}^{T} & -\mathbf{Z}_{2}
\end{array}\right]<\mathbf{0}
$$

where

$$
\begin{aligned}
& \boldsymbol{\Psi}=\boldsymbol{\Lambda}_{1}^{T} \boldsymbol{\Phi}_{1} \boldsymbol{\Lambda}_{1}+\boldsymbol{\Lambda}_{2}^{T} \boldsymbol{\Phi}_{2} \boldsymbol{\Lambda}_{2}+\Lambda_{3}^{T} \boldsymbol{\Phi}_{3} \Lambda_{3}-\Lambda_{4}^{T} \mathbf{Q}_{2} \Lambda_{4}+\boldsymbol{\Lambda}_{1}^{T} \boldsymbol{\Phi}_{4} \Lambda_{3}+\Lambda_{3}^{T} \boldsymbol{\Phi}_{4}^{T} \boldsymbol{\Lambda}_{1}-\Lambda_{5}^{T} \mathbf{Z}_{1} \Lambda_{5}
\end{aligned}
$$

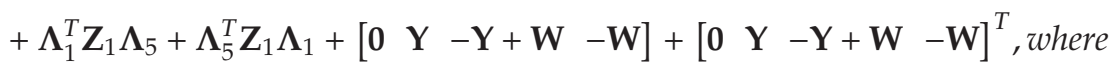

$$
\begin{aligned}
& \boldsymbol{\Lambda}_{1}=\left[\begin{array}{ll}
\mathbf{I}_{n} & \mathbf{0}_{n \times(m+2) n}
\end{array}\right] \text {, }
\end{aligned}
$$




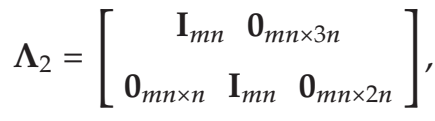

$$
\begin{aligned}
& \Lambda_{3}=\left[\begin{array}{lll}
\mathbf{0}_{n \times(m+1) n} & \mathbf{I}_{n} & \mathbf{0}_{n}
\end{array}\right] \text {, } \\
& \Lambda_{4}=\left[\begin{array}{ll}
\mathbf{0}_{n \times(m+2) n} & \mathbf{I}_{n}
\end{array}\right] \text {, } \\
& \boldsymbol{\Lambda}_{5}=\left[\begin{array}{lll}
\mathbf{0}_{n} & \mathbf{I}_{n} & \mathbf{0}_{n \times(m+1) n}
\end{array}\right], \\
& \mathbf{\Phi}_{1}=\mathbf{A}^{T} \mathbf{P A}-\mathbf{P}+\mathbf{Q}_{2}+\left(h_{2}-\tau m+1\right) \mathbf{Q}_{3}-\mathbf{Z}_{1}+\left(\mathbf{A}-\mathbf{I}_{n}\right)^{T}\left(\tau^{2} \mathbf{Z}_{1}+\left(h_{2}-\tau m\right)^{2} \mathbf{Z}_{2}\right)\left(\mathbf{A}-\mathbf{I}_{n}\right), \\
& \mathbf{\Phi}_{2}=\left[\begin{array}{cc}
\mathbf{Q}_{1} & \mathbf{0} \\
\mathbf{0} & -\mathbf{Q}_{1}
\end{array}\right] \\
& \mathbf{\Phi}_{3}=\mathbf{A}_{1}^{T}\left(\mathbf{P}+\tau^{2} \mathbf{Z}_{1}+\left(h_{2}-\tau m\right)^{2} \mathbf{Z}_{2}\right) \mathbf{A}_{1}-\mathbf{Q}_{3} \\
& \mathbf{\Phi}_{4}=\mathbf{A}^{T} \mathbf{P} \mathbf{A}_{1}+\left(\mathbf{A}-\mathbf{I}_{n}\right)^{T}\left(\tau^{2} \mathbf{Z}_{1}+\left(h_{2}-\tau m\right)^{2} \mathbf{Z}_{2}\right) \mathbf{A}_{1} .
\end{aligned}
$$

Proof. Choose a Lyapunov functional candidate as

$$
\begin{aligned}
V(k)= & \mathbf{x}^{T}(k) \mathbf{P} \mathbf{x}(k)+\sum_{i=k-\tau}^{k-1} \mathbf{\Upsilon}^{T}(i) \mathbf{Q}_{1} \mathbf{\Upsilon}(i)+\sum_{i=k-h_{2}}^{k-1} \mathbf{x}^{T}(i) \mathbf{Q}_{2} \mathbf{x}(i)+\sum_{j=-h_{2}} \sum_{i=k+j}^{-\tau m} \mathbf{x}^{T}(i) \mathbf{Q}_{3} \mathbf{x}(i) \\
& +\sum_{j=-\tau}^{-1} \sum_{i=k+j}^{k-1} \tau \Delta \mathbf{x}^{T}(i) \mathbf{Z}_{1} \Delta \mathbf{x}(i)+\sum_{j=-h_{2}}^{-\tau m-1} \sum_{i=k+j}^{k-1}\left(h_{2}-\tau m\right) \Delta \mathbf{x}^{T}(i) \mathbf{Z}_{2} \Delta \mathbf{x}(i),
\end{aligned}
$$

where

$$
\begin{aligned}
& \boldsymbol{\Upsilon}(i)=\left[\begin{array}{llll}
\mathbf{x}^{T}(i) & \mathbf{x}^{T}(i-\tau) & \cdots & \mathbf{x}^{T}(i-(m-1) \tau)
\end{array}\right]^{T}, \\
& \Delta \mathbf{x}(i)=\mathbf{x}(i+1)-\mathbf{x}(i)
\end{aligned}
$$

Taking the forward difference of (3.3) along the solution of (2.1a) and (2.1b), we have

$$
\begin{aligned}
\Delta V(k)= & V(k+1)-V(k) \\
= & {\left[\mathbf{A x}(k)+\mathbf{A}_{1} \mathbf{x}(k-d(k))\right]^{T} \mathbf{P}\left[\mathbf{A x}(k)+\mathbf{A}_{1} \mathbf{x}(k-d(k))\right]-\mathbf{x}^{T}(k) \mathbf{P} \mathbf{x}(k) } \\
& +\boldsymbol{\Upsilon}^{T}(k) \mathbf{Q}_{1} \mathbf{\Upsilon}(k)-\mathbf{\Upsilon}^{T}(k-\tau) \mathbf{Q}_{1} \mathbf{\Upsilon}(k-\tau)+\mathbf{x}^{T}(k) \mathbf{Q}_{2} \mathbf{x}(k) \\
& -\mathbf{x}^{T}\left(k-h_{2}\right) \mathbf{Q}_{2} \mathbf{x}\left(k-h_{2}\right)+\left(h_{2}-\tau m+1\right) \mathbf{x}^{T}(k) \mathbf{Q}_{3} \mathbf{x}(k)
\end{aligned}
$$




$$
\begin{aligned}
& -\sum_{i=k-h_{2}}^{k-\tau m} \mathbf{x}^{T}(i) \mathbf{Q}_{3} \mathbf{x}(i)+\Delta \mathbf{x}^{T}(k)\left(\tau^{2} \mathbf{Z}_{1}+\left(h_{2}-\tau m\right)^{2} \mathbf{Z}_{2}\right) \Delta \mathbf{x}(k) \\
& -\sum_{i=k-\tau}^{k-1} \tau \Delta \mathbf{x}^{T}(i) \mathbf{Z}_{1} \Delta \mathbf{x}(i)-\sum_{i=k-h_{2}}^{k-\tau m-1}\left(h_{2}-\tau m\right) \Delta \mathbf{x}^{T}(i) \mathbf{Z}_{2} \Delta \mathbf{x}(i) .
\end{aligned}
$$

Using Lemma 2.2, we obtain

$$
-\sum_{i=k-\tau}^{k-1} \tau \Delta \mathbf{x}^{T}(i) \mathbf{Z}_{1} \Delta \mathbf{x}(i) \leq-(\mathbf{x}(k)-\mathbf{x}(k-\tau))^{T} \mathbf{Z}_{1}(\mathbf{x}(k)-\mathbf{x}(k-\tau))
$$

Note that

$$
-\sum_{i=k-h_{2}}^{k-\tau m} \mathbf{x}^{T}(i) \mathbf{Q}_{3} \mathbf{x}(i) \leq-\mathbf{x}^{T}(k-d(k)) \mathbf{Q}_{3} \mathbf{x}(k-d(k))
$$

Now, we have the following relations:

$$
\begin{aligned}
& 0=2 \zeta^{T}(k) \mathbf{Y}\left[\mathbf{x}(k-\tau m)-\mathbf{x}(k-d(k))-\sum_{i=k-d(k)}^{k-\tau m-1} \Delta \mathbf{x}(i)\right], \\
& 0=2 \zeta^{T}(k) \mathbf{W}\left[\mathbf{x}(k-d(k))-\mathbf{x}\left(k-h_{2}\right)-\sum_{i=k-h_{2}}^{k-d(k)-1} \Delta \mathbf{x}(i)\right]
\end{aligned}
$$

where $\mathbf{Y}$ and $\mathbf{W}$ are constant matrices of appropriate dimensions and

$$
\zeta(k)=\left[\begin{array}{llll}
\mathbf{\Upsilon}^{T}(k) & \mathbf{x}^{T}(k-\tau m) & \mathbf{x}^{T}(k-d(k)) & \mathbf{x}^{T}\left(k-h_{2}\right)
\end{array}\right]^{T} .
$$

It follows from (2.1a) and (3.5) that

$$
\Delta \mathbf{x}(k)=\left(\mathbf{A}-\mathbf{I}_{n}\right) \mathbf{x}(k)+\mathbf{A}_{1} \mathbf{x}(k-d(k)) .
$$

Employing (3.6)-(3.11), we have the following inequality:

$$
\begin{aligned}
\Delta V(k) \leq & \zeta^{T}(k) \Psi \zeta(k)-2 \zeta^{T}(k) \mathbf{Y} \sum_{i=k-d(k)}^{k-\tau m-1} \Delta \mathbf{x}(i)-2 \zeta^{T}(k) \mathbf{W} \sum_{i=k-h_{2}}^{k-d(k)-1} \Delta \mathbf{x}(i) \\
& -\sum_{i=k-d(k)}^{k-\tau m-1}\left(h_{2}-\tau m\right) \Delta \mathbf{x}^{T}(i) \mathbf{Z}_{2} \Delta \mathbf{x}(i)-\sum_{i=k-h_{2}}^{k-d(k)-1}\left(h_{2}-\tau m\right) \Delta \mathbf{x}^{T}(i) \mathbf{Z}_{2} \Delta \mathbf{x}(i),
\end{aligned}
$$


where $\Psi$ is given by (3.2). Equation (3.12) can also be rearranged as

$$
\begin{aligned}
\Delta V(k) \leq & \frac{1}{\left(h_{2}-\tau m\right)} \sum_{i=k-d(k)}^{k-\tau m-1}\left[\begin{array}{c}
\zeta(k) \\
-\left(h_{2}-\tau m\right) \Delta \mathbf{x}(i)
\end{array}\right]^{T}\left[\begin{array}{cc}
\Psi & \mathbf{Y} \\
\mathbf{Y}^{T} & -\mathbf{Z}_{2}
\end{array}\right]\left[\begin{array}{c}
\zeta(k) \\
-\left(h_{2}-\tau m\right) \Delta \mathbf{x}(i)
\end{array}\right] \\
& +\frac{1}{\left(h_{2}-\tau m\right)} \sum_{i=k-h_{2}}^{k-d(k)-1}\left[\begin{array}{c}
\zeta(k) \\
-\left(h_{2}-\tau m\right) \Delta \mathbf{x}(i)
\end{array}\right]^{T}\left[\begin{array}{cc}
\boldsymbol{\Psi} & \mathbf{W} \\
\mathbf{W}^{T} & -\mathbf{Z}_{2}
\end{array}\right]\left[\begin{array}{c}
\zeta(k) \\
-\left(h_{2}-\tau m\right) \Delta \mathbf{x}(i)
\end{array}\right] .
\end{aligned}
$$

Using [17, Lemma 4.1], it can be shown that there exists a matrix $\mathbf{X}$ of appropriate dimensions satisfying (3.1) if and only if

$$
\left[\begin{array}{cc}
\Psi & \mathbf{Y} \\
\mathbf{Y}^{T} & -\mathbf{Z}_{2}
\end{array}\right]<\mathbf{0}, \quad\left[\begin{array}{cc}
\Psi & \mathbf{W} \\
\mathbf{W}^{T} & -\mathbf{Z}_{2}
\end{array}\right]<\mathbf{0},
$$

which together with (3.13) implies $\Delta V(k)<0$ for all nonzero $\zeta(k)$. This completes the proof.

Remark 3.2. A comparison of the number of the decision variables involved in several recent stability results is summarized in Table 1. It may be observed that the size of complexity in $[22,26,31]$ is only related to state dimension $n$, whereas the complexity of [37] and Theorem 3.1 depends on both $n$ and $m$.

The total number of scalar decision variables of Theorem 3.1 is $D_{1}=(n / 2)\left[n\left(m^{2}+4 m+\right.\right.$ $19)+(m+5)]$, and the total row size of the LMIs is $L_{1}=2 n(m+5)$. The numerical complexity of Theorem 3.1 is proportional to $L_{1} D_{1}^{3}$ [16]. In [37], the total number of scalar decision variables of Theorem 2 is $D_{2}=(n / 2)\left[n\left(3 m^{2}+18 m+41\right)+(3 m+11)\right]$, the total row size of the LMIs is $L_{2}=n(7 m+31)$, and the numerical complexity is proportional to $L_{2} D_{2}^{3}$. Therefore, Theorem 3.1 has much smaller numerical complexity than Theorem 2 of [37].

Remark 3.3. For a given $h_{1}$, the allowable maximum value of $h_{2}$ for guaranteeing the asymptotic stability of system (2.1a) and (2.1b)-(2.3) can be obtained by iteratively solving the LMI (3.1).

Remark 3.4. With $m=1, \mathbf{Y}=\left[\begin{array}{lll}\mathbf{Y}_{1}^{T} & \mathbf{Y}_{2}^{T} & \mathbf{0}_{n \times 2 n}\end{array}\right]^{T}$, and $\mathbf{W}=\left[\begin{array}{lll}\mathbf{W}_{1}^{T} & \mathbf{W}_{2}^{T} & \mathbf{0}_{n \times 2 n}\end{array}\right]^{T}$, Theorem 3.1 reduces to an equivalent form of [26, Proposition 1]. Thus, as compared to [26, Proposition 1], Theorem 3.1 provides additional degrees of freedom in the selection of $m, \mathbf{Y}$, and $\mathbf{W}$ which would result in an enhanced stability region in the parameter space.

Remark 3.5. Using similar steps as in the proof of [37, Proposition 8], it is easy to establish that the conservatism of the stability result obtained via Theorem 3.1 is nonincreasing as the number of partitions increases.

It may be noted that, in the derivation of Theorem 3.1, the lower bound of the delay is assumed to be $h_{1}=\tau m \geq 1$. For the situation where $h_{1}=0$, we have the following result. 
Table 1: Comparison of the number of decision variables involved in various methods.

\begin{tabular}{lc}
\hline Methods & Number of the decision variables \\
\hline Theorem 1 in [22] & $\left(23 n^{2}+5 n\right) / 2$ \\
Theorem 3 in [22] & $\left(67 n^{2}+9 n\right) / 2$ \\
Theorem 1 in [31] & $9 n^{2}+3 n$ \\
Theorem 1 in [23] & $13 n^{2}+5 n$ \\
Proposition 1 in [26] & $8 n^{2}+3 n$ \\
Theorem 2 in [37] & $(n / 2)\left[n\left(3 m^{2}+18 m+41\right)+(3 m+11)\right]$ \\
Theorem 3.1 & $(n / 2)\left[n\left(m^{2}+4 m+19\right)+(m+5)\right]$ \\
\hline
\end{tabular}

Theorem 3.6. The system (2.1a) and (2.1b) with $0 \leq d(k) \leq h_{2}$ is asymptotically stable if there exist real matrices $\mathbf{P}=\mathbf{P}^{T}>\mathbf{0}, \mathbf{Q}_{i}=\mathbf{Q}_{i}^{T}>\mathbf{0}(i=1,2), \mathbf{Z}=\mathbf{Z}^{T}>\mathbf{0}, \mathbf{X}, \widetilde{\mathbf{Y}}$, and $\widetilde{\mathbf{W}}$ such that

$$
\left[\begin{array}{ccc}
-\mathbf{Z} & \tilde{\mathbf{Y}}^{T} & \mathbf{X} \\
\tilde{\mathbf{Y}} & \widetilde{\boldsymbol{\Psi}} & \widetilde{\mathbf{W}} \\
\mathbf{X}^{T} & \widetilde{\mathbf{W}}^{T} & -\mathbf{Z}
\end{array}\right]<\mathbf{0}
$$

where

$$
\begin{aligned}
& \widetilde{\mathbf{\Psi}}=\tilde{\mathbf{\Lambda}}_{1}^{T} \widetilde{\mathbf{\Phi}}_{1} \tilde{\mathbf{\Lambda}}_{1}+\tilde{\mathbf{\Lambda}}_{1}^{T} \widetilde{\mathbf{\Phi}}_{2} \tilde{\mathbf{\Lambda}}_{2}+\tilde{\mathbf{\Lambda}}_{2}^{T} \tilde{\mathbf{\Phi}}_{2}^{T} \tilde{\mathbf{\Lambda}}_{1}+\tilde{\mathbf{\Lambda}}_{2}^{T} \widetilde{\mathbf{\Phi}}_{3} \tilde{\mathbf{\Lambda}}_{2}-\tilde{\mathbf{\Lambda}}_{3}^{T} \mathbf{Q}_{1} \tilde{\mathbf{\Lambda}}_{3} \\
& +[\tilde{\mathbf{Y}}-\tilde{\mathbf{Y}}+\widetilde{\mathbf{W}}-\widetilde{\mathbf{W}}]+[\tilde{\mathbf{Y}}-\tilde{\mathbf{Y}}+\widetilde{\mathbf{W}}-\widetilde{\mathbf{W}}]^{T} \text {, where } \\
& \tilde{\Lambda}_{1}=\left[\begin{array}{ll}
\mathbf{I}_{n} & \mathbf{0}_{n \times 2 n}
\end{array}\right] \text {, } \\
& \tilde{\Lambda}_{2}=\left[\begin{array}{lll}
\mathbf{0}_{n} & \mathbf{I}_{n} & \mathbf{0}_{n}
\end{array}\right] \text {, } \\
& \tilde{\Lambda}_{3}=\left[\begin{array}{ll}
\mathbf{0}_{n \times 2 n} & \mathbf{I}_{n}
\end{array}\right] \text {, } \\
& \widetilde{\mathbf{\Phi}}_{1}=\mathbf{A}^{T} \mathbf{P A}-\mathbf{P}+h_{2}{ }^{2}\left(\mathbf{A}-\mathbf{I}_{n}\right)^{T} \mathbf{Z}\left(\mathbf{A}-\mathbf{I}_{n}\right)+\mathbf{Q}_{1}+\left(h_{2}+1\right) \mathbf{Q}_{2}, \\
& \widetilde{\mathbf{\Phi}}_{2}=\mathbf{A}^{T} \mathbf{P} \mathbf{A}_{1}+h_{2}{ }^{2}\left(\mathbf{A}-\mathbf{I}_{n}\right)^{T} \mathbf{Z} \mathbf{A}_{1} \text {, } \\
& \widetilde{\mathbf{\Phi}}_{3}=\mathbf{A}_{1}^{T}\left(\mathbf{P}+h_{2}^{2} \mathbf{Z}\right) \mathbf{A}_{1}-\mathbf{Q}_{2} \text {. }
\end{aligned}
$$

Proof. Choosing the Lyapunov functional as

$$
\begin{aligned}
V(k)= & \mathbf{x}^{T}(k) \mathbf{P} \mathbf{x}(k)+\sum_{i=k-h_{2}}^{k-1} \mathbf{x}^{T}(i) \mathbf{Q}_{1} \mathbf{x}(i) \\
& +\sum_{j=-h_{2}}^{0} \sum_{i=k+j}^{k-1} \mathbf{x}^{T}(i) \mathbf{Q}_{2} \mathbf{x}(i)+\sum_{j=-h_{2}}^{-1} \sum_{i=k+j}^{k-1} h_{2} \Delta \mathbf{x}^{T}(i) \mathbf{Z} \Delta \mathbf{x}(i)
\end{aligned}
$$

and employing the steps of the proof of Theorem 3.1 discussed earlier, one can easily arrive at the above theorem. The details of the proof of Theorem 3.6 are, therefore, omitted. 
Table 2: Admissible upper bound of $h_{2}$ for various $h_{1}$.

\begin{tabular}{lcc}
\hline Method & $h_{1}=4$ & $h_{1}=12$ \\
\hline Theorem 1 in [29] & 8 & 13 \\
Theorem 1 in [22] & 13 & 16 \\
Theorem 1 in [23] & 17 & 21 \\
Proposition 1 in [26] & 17 & 21 \\
& & \\
Theorem 2 in [37] & $17(m=2, \tau=2)$ & $21(m=1, \tau=12)$ \\
& $18(m=4, \tau=1)$ & $22(m=2, \tau=6)$ \\
Theorem 3.1 & $17(m=2, \tau=2)$ & $21(m=1, \tau=12)$ \\
& $18(m=4, \tau=1)$ & $22(m=2, \tau=6)$ \\
\hline
\end{tabular}

Remark 3.7. With $\widetilde{L}_{1}=9 n$ rows and $\widetilde{D}_{1}=9 n^{2}+2 n$ scalar decision variables, LMI (3.15) in Theorem 3.6 has the numerical complexity proportional to $\widetilde{L}_{1} \widetilde{D}_{1}^{3}[16]$, while the condition in [37, Proposition 10] has the numerical complexity proportional to $\widetilde{L}_{2} \widetilde{D}_{2}^{3}$, where $\widetilde{L}_{2}=21 n$ and $\widetilde{D}_{2}=(n / 2)(25 n+7)$. So, Theorem 3.6 has smaller numerical complexity than [37, Proposition 10].

\section{A Numerical Example}

To demonstrate the applicability of the presented results and compare them with previous results, we now consider a specific example of system (2.1a), (2.1b) with

$$
\mathbf{A}=\left[\begin{array}{cc}
0.8 & 0 \\
0.05 & 0.9
\end{array}\right], \quad \mathbf{A}_{1}=\left[\begin{array}{cc}
-0.1 & 0 \\
-0.2 & -0.1
\end{array}\right]
$$

and $0 \leq d(k) \leq h_{2}$. This example has been considered in [22, 29, 37]. Using Matlab LMI toolbox $[38,39]$, it is found from Theorem 3.6 that the present system is stable for $0 \leq d(k) \leq$ 17. In this case, the number of decision variables involved in Theorem 3.6 is 40 . On the other hand, to obtain the upper bound $h_{2}=17$, [37, Proposition 10] requires 57 decision variables. This demonstrates the numerical efficiency of the proposed method.

Next, consider the system described by (2.1a), (2.1b)-(2.3), and (4.1). For different values of $h_{1}$, the admissible upper bound $h_{2}$ is listed in Table 2. From Table 2, it is clear that Theorem 3.1 can provide a larger upper bound $h_{2}$ than the previously reported stability results $[22,23,26,29]$. It may also be observed that, for the example under consideration, Theorem 3.1 leads to upper bound $h_{2}$ which is identical to that arrived at via Theorem 2 in [37]. However, as discussed in Remark 3.2, Theorem 3.1 has much smaller numerical complexity than Theorem 2 in [37].

\section{Conclusion}

In this paper, the problem concerning the asymptotic stability of linear discrete-time systems with interval-like time-varying delay in the state has been considered. Using the concept of delay partitioning, an LMI-based criterion for the asymptotic stability of such systems has 
been established. The criterion depends on the size of delay as well as partition size. The presented approach may imply asymptotic stability for a broader class of time-varying statedelayed systems, as compared to previous approaches [22, 23, 26, 29]. The presented criterion is numerically less complex than [37]. The stability results discussed in this paper can easily be extended to delayed discrete-time systems with parameter uncertainties.

\section{References}

[1] J. K. Hale, Functional Differential Equations, Springer, New York, NY, USA, 1971.

[2] H. Huang and G. Feng, "Improved approach to delay-dependent stability analysis of discrete-time systems with time-varying delay," IET Control Theory $\mathcal{E}$ Applications, vol. 4, no. 10, pp. 2152-2159, 2010.

[3] J.-P. Richard, "Time-delay systems: an overview of some recent advances and open problems," Automatica, vol. 39, no. 10, pp. 1667-1694, 2003.

[4] H. Shao, "Delay-dependent approaches to globally exponential stability for recurrent neural networks," IEEE Transactions on Circuits and Systems II, vol. 55, no. 6, pp. 591-595, 2008.

[5] H. Shao, "Delay-dependent stability for recurrent neural networks with time-varying delays," IEEE Transactions on Neural Networks, vol. 19, no. 9, pp. 1647-1651, 2008.

[6] V. J. S. Leite and M. F. Miranda, "Robust stabilization of discrete-time systems with time-varying delay: an LMI approach," Mathematical Problems in Engineering, vol. 2008, Article ID 875609, 15 pages, 2008.

[7] S. Xu and J. Lam, "A survey of linear matrix inequality techniques in stability analysis of delay systems," International Journal of Systems Science, vol. 39, no. 12, pp. 1095-1113, 2008.

[8] S. F. Chen, "Asymptotic stability of discrete-time systems with time-varying delay subject to saturation nonlinearities," Chaos, Solitons and Fractals, vol. 42, no. 2, pp. 1251-1257, 2009.

[9] M. S. Mahmoud, "Robust stability and stabilization of a class of uncertain nonlinear systems with delays," Mathematical Problems in Engineering, vol. 4, no. 2, pp. 165-185, 1998.

[10] M. S. Mahmoud, F. M. Al-Sunni, and Y. Shi, "Switched discrete-time delay systems: delay-dependent analysis and synthesis," Circuits, Systems, and Signal Processing, vol. 28, no. 5, pp. 735-761, 2009.

[11] M. S. Mahmoud and Y. Xia, "Robust stability and stabilization of a class of nonlinear switched discrete-time systems with time-varying delays," Journal of Optimization Theory and Applications, vol. 143, no. 2, pp. 329-355, 2009.

[12] S. Xu and J. Lam, "On equivalence and efficiency of certain stability criteria for time-delay systems," IEEE Transactions on Automatic Control, vol. 52, no. 1, pp. 95-101, 2007.

[13] E. Fridman and U. Shaked, "A descriptor system approach to $H_{\infty}$ control of linear time-delay systems," IEEE Transactions on Automatic Control, vol. 47, no. 2, pp. 253-270, 2002.

[14] Q.-L. Han, "On stability of linear neutral systems with mixed time delays: a discretized Lyapunov functional approach," Automatica, vol. 41, no. 7, pp. 1209-1218, 2005.

[15] Q.-L. Han, "A discrete delay decomposition approach to stability of linear retarded and neutral systems," Automatica, vol. 45, no. 2, pp. 517-524, 2009.

[16] Q.-L. Han, "Improved stability criteria and controller design for linear neutral systems," Automatica, vol. 45, no. 8, pp. 1948-1952, 2009.

[17] Q.-L. Han and K. Gu, "Stability of linear systems with time-varying delay: a generalized discretized lyapunov functional approach," Asian Journal of Control, vol. 3, no. 3, pp. 170-180, 2001.

[18] X. Jiang and Q.-L. Han, "On $H_{\infty}$ control for linear systems with interval time-varying delay," Automatica, vol. 41, no. 12, pp. 2099-2106, 2005.

[19] M. Wu, Y. He, J.-H. She, and G.-P. Liu, “Delay-dependent criteria for robust stability of time-varying delay systems," Automatica, vol. 40, no. 8, pp. 1435-1439, 2004.

[20] H. R. Karimi, M. Zapateiro, and N. Luo, "New delay-dependent stability criteria for uncertain neutral systems with mixed time-varying delays and nonlinear perturbations," Mathematical Problems in Engineering, vol. 2009, Article ID 759248, 22 pages, 2009.

[21] B. Du and X. Zhang, "Delay-dependent stability analysis and synthesis for uncertain impulsive switched system with mixed delays," Discrete Dynamics in Nature and Society, vol. 2011, Article ID 381571, 9 pages, 2011.

[22] H. Gao and T. Chen, "New results on stability of discrete-time systems with time-varying state delay," IEEE Transactions on Automatic Control, vol. 52, no. 2, pp. 328-334, 2007. 
[23] Y. He, M. Wu, G.-P. Liu, and J.-H. She, “Output feedback stabilization for a discrete-time system with a time-varying delay," IEEE Transactions on Automatic Control, vol. 53, no. 10, pp. 2372-2377, 2008.

[24] V. K. R. Kandanvli and H. Kar, "Delay-dependent LMI condition for global asymptotic stability of discrete-time uncertain state-delayed systems using quantization/overflow nonlinearities," International Journal of Robust and Nonlinear Control, vol. 21, no. 14, pp. 1611-1622, 2011.

[25] H. Shao, "New delay-dependent stability criteria for systems with interval delay," Automatica, vol. 45, no. 3, pp. 744-749, 2009.

[26] H. Shao and Q.-L. Han, "New stability criteria for linear discrete-time systems with interval-like timevarying delays," IEEE Transactions on Automatic Control, vol. 56, no. 3, pp. 619-625, 2011.

[27] K. Ratchagit and V. N. Phat, "Stability criterion for discrete-time systems," journal of Inequalities and Applications, vol. 2010, Article ID 201459, 6 pages, 2010.

[28] E. Fridman and U. Shaked, "Stability and guaranteed cost control of uncertain discrete delay systems," International Journal of Control, vol. 78, no. 4, pp. 235-246, 2005.

[29] H. Gao, J. Lam, C. Wang, and Y. Wang, "Delay-dependent output-feedback stabilisation of discretetime systems with time-varying state delay," IET Control Theory and Applications, vol. 151, no. 6, pp. 691-698, 2004.

[30] X. Jiang, Q.-L. Han, and X. Yu, "Stability criteria for linear discrete-time systems with interval-like time-varying delay," in Proceedings of the American Control Conference, pp. 2817-2822, Portland, Ore, USA, June 2005.

[31] B. Zhang, S. Xu, and Y. Zou, "Improved stability criterion and its applications in delayed controller design for discrete-time systems," Automatica, vol. 44, no. 11, pp. 2963-2967, 2008.

[32] H. Huang and G. Feng, "State estimation of recurrent neural networks with time-varying delay: a novel delay partition approach," Neurocomputing, vol. 74, no. 5, pp. 792-796, 2011.

[33] B. Du, J. Lam, Z. Shu, and Z. Wang, "A delay-partitioning projection approach to stability analysis of continuous systems with multiple delay components," IET Control Theory and Applications, vol. 3, no. 4, pp. 383-390, 2009.

[34] Y. Zhao, H. Gao, J. Lam, and B. Du, "Stability and stabilization of delayed T-S fuzzy systems: a delay partitioning approach," IEEE Transactions on Fuzzy Systems, vol. 17, no. 4, pp. 750-762, 2009.

[35] J. Liu, B. Yao, and Z. Gu, "Delay-dependent $H_{\infty}$ filtering for Markovian jump time-delay systems: a piecewise analysis method," Circuits Systems and Signal Processing, vol. 30, no. 6, pp. 1253-1273, 2011.

[36] L. Wu, J. Lam, X. Yao, and J. Xiong, "Robust guaranteed cost control of discrete-time networked control systems," Optimal Control Applications and Methods, vol. 32, no. 1, pp. 95-112, 2011.

[37] X. Meng, J. Lam, B. Du, and H. Gao, "A delay-partitioning approach to the stability analysis of discrete-time systems," Automatica, vol. 46, no. 3, pp. 610-614, 2010.

[38] S. Boyd, L. El Ghaoui, E. Feron, and V. Balakrishnan, Linear Matrix Inequalities in System and Control Theory, vol. 15 of SIAM Studies in Applied Mathematics, Society for Industrial and Applied Mathematics (SIAM), Philadelphia, Pa, USA, 1994.

[39] P. Gahinet, A. Nemirovski, A. J. Laub, and M. Chilali, LMI Control Toolbox for Use With Matlab, The MATH Works, Natick, Mass, USA, 1995. 


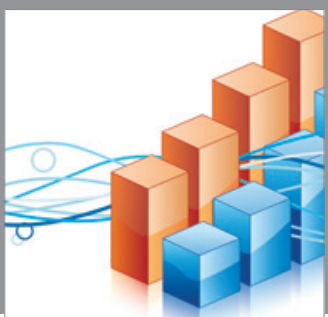

Advances in

Operations Research

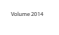

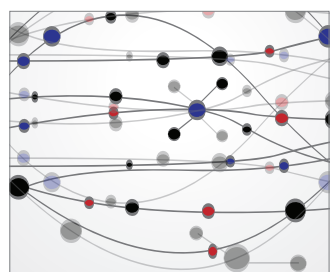

\section{The Scientific} World Journal
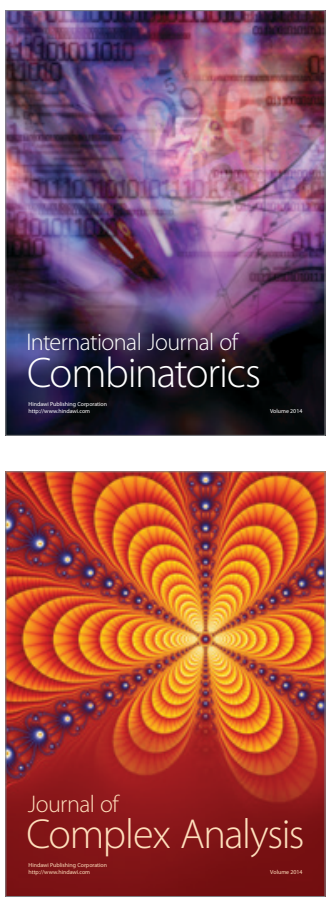

International Journal of

Mathematics and

Mathematical

Sciences
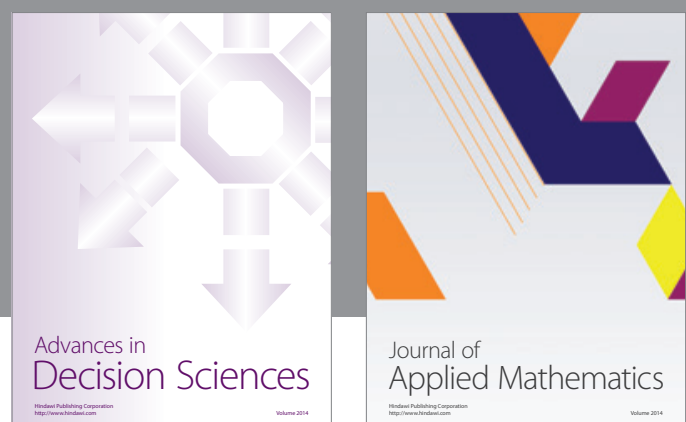

Journal of

Applied Mathematics
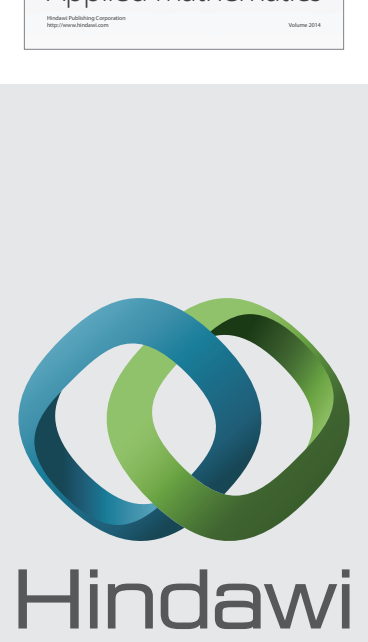

Submit your manuscripts at http://www.hindawi.com
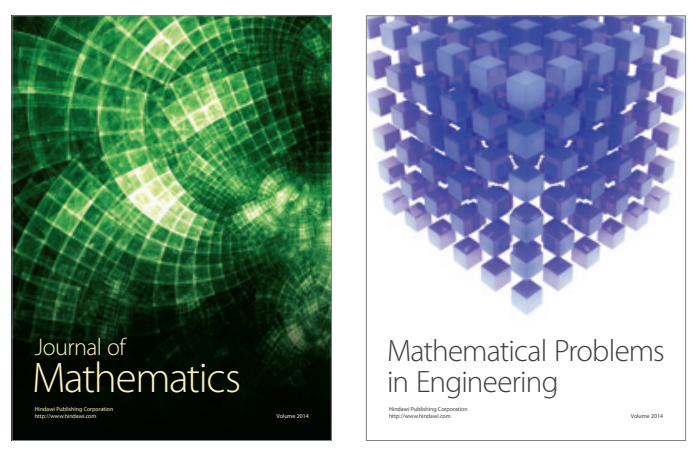

Mathematical Problems in Engineering
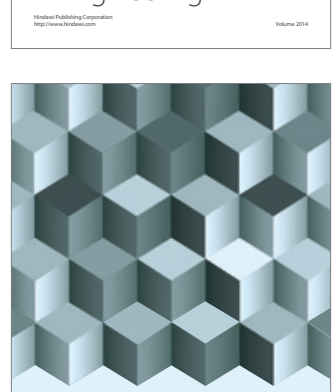

Journal of

Function Spaces
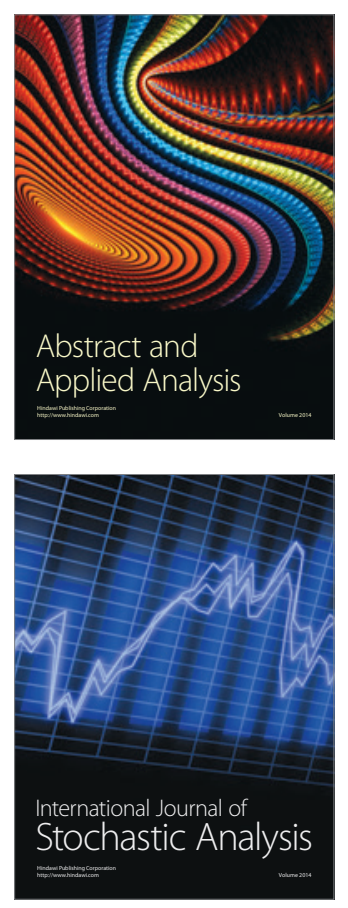

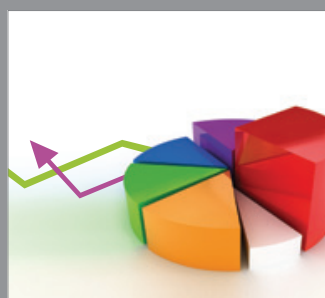

ournal of

Probability and Statistics

Promensencen
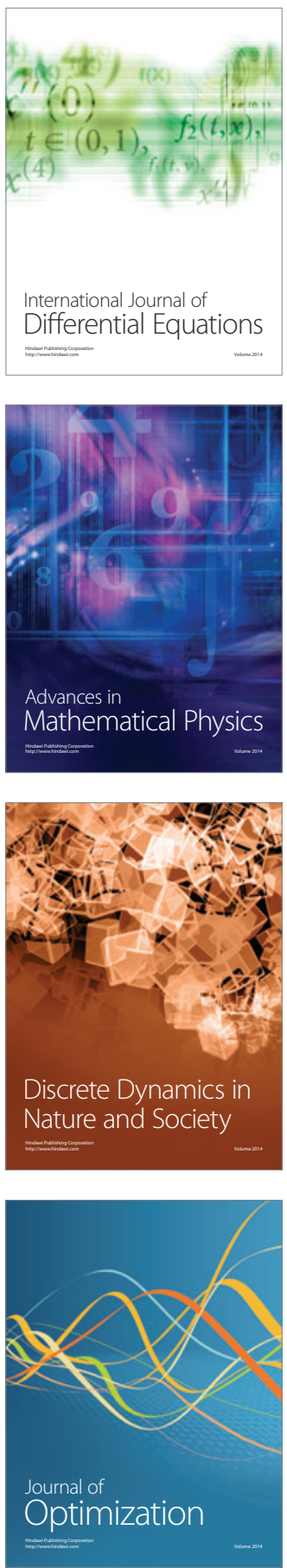